\title{
Leiomyosarcoma of Inferior Vena Cava Arising from the Confluence of Right Adrenal Vein Masquerading as a Suprarenal Mass - A Case Report
}

\section{Rakesh Manilal Sharma ${ }^{1}$, Sandeep Maheswara Reddy Kallam ${ }^{2 *}$, B Vishal Rao ${ }^{3}$ and Ashwin Giridhar ${ }^{4}$}

${ }^{1}$ Consultant Uro-Oncologist, Division of Uro-Oncology, Department of Surgical

Oncology, Basavatarakam Indo American Cancer Hospital and Research Institute,

Hyderabad, India

${ }^{2}$ Fellow in Uro-Oncology, Division of Uro-Oncology, Department of Surgical Oncol-

ogy, Basavatarakam Indo American Cancer Hospital and Research Institute,

Hyderabad, India

${ }^{3}$ Consultant Pathologist, Department of Pathology, Basavatarakam Indo American

Cancer Hospital and Research Institute, Hyderabad, India

${ }^{4}$ Consultant Uro-Oncologist, Division of Uro-Oncology, Department of Surgical

Oncology, Basavatarakam Indo American Cancer Hospital and Research Institute,

Hyderabad, India

*Corresponding Author: Sandeep Maheswara Reddy Kallam, Fellow in Uro-

Oncology, Division of Uro-Oncology, Department of Surgical Oncology, Basavatarakam

Indo American Cancer Hospital and Research Institute, Hyderabad, India.
Received: January 21, 2021

Published: February 26, 2021

(C) All rights are reserved by Sandeep

Maheswara Reddy Kallam., et al.

\begin{abstract}
Leiomyosarcoma (LMS) is a malignant mesenchymal tumour, which originates from the smooth muscle cells. Leiomyosarcoma of vascular origin most commonly originates from inferior vena cava (IVC). Around 400 cases of IVC leiomyosarcoma were reported in literature till date. Here we report a case of a 58-year-old male diagnosed to have right large palpable supra renal mass. Contrast enhanced computed tomography (CECT) of abdomen and pelvis revealed a well-defined soft tissue mass measuring around $10 \mathrm{cms}$ with heterogeneous post contrast enhancement at right suprarenal region with a clinical differential diagnosis of adreno cortical carcinoma and retroperitoneal leiomyosarcoma was made. Intra-operatively right adrenal gland was normal, and the mass was seen arising from the side wall of IVC at the confluence of right adrenal vein. Complete excision of mass done, followed by repair of IVC rent. Histopathological examination showed leiomyosarcoma. Adrenal gland was normal, margins were free of tumour and immunohistochemistry (IHC) was positive for caldesmon and smooth muscle actin (SMA). Patient was on follow up for the past 1 year without local recurrence or distant metastasis.
\end{abstract}

Keywords: IVC Leiomyosarcoma; Vascular LMS; Suprarenal Mass Differential Diagnosis; Inferior Vena Cava Tumour; Caldesmon; Smooth Muscle Actin

\section{Abbreviations}

LMS: Leiomyosarcoma; IVC: Inferior Vena Cava; IHC: Immunohistochemistry; SMA: Smooth Muscle Actin; CECT: Contrast Enhanced Computed Tomography; CT: Computed Tomography; H\&E: Hematoxylin and Eosin.

\section{Introduction}

Leiomyosarcoma is a soft-tissue malignancy that shows smooth muscle differentiation. Primary leiomyosarcomas of vascular origin are relatively rare and vascular LMS frequently arises from IVC 
$[1,2]$. Rarely, leiomyosarcoma may arise from the renal vein or adrenal vein and extremely rarely from adrenal gland [3]. Here we are reporting a case of Leiomyosarcoma of the IVC arising at the confluence of right adrenal vein draining in to IVC presenting as right suprarenal mass.

\section{Case Report}

A 58-year-old male presented with chief complaints of intermittent fever. Palpable abdominal mass was noted in right lumbar region and epigastrium on physical examination. On sonography of abdomen a large well defined heterogeneous lesion of size 9.6 x $7.9 \mathrm{cms}$ was seen in right supra renal area with hypoechoic and hyperechoic areas without definite vascularity in it.

Contrast enhanced computed tomography (CECT) of abdomen and pelvis revealed a well-defined round to oval, soft tissue mass measuring 9.7 x $8.4 \mathrm{cms}$ with heterogeneous post contrast enhancement at right suprarenal region with mass causing anteromedial displacement of IVC and downward displacement of right kidney and in close contact with right lobe of liver with no evidence of haemorrhage, fat, calcific densities, or venous thrombosis (Figure 1 and 2). CT chest was within normal limits.

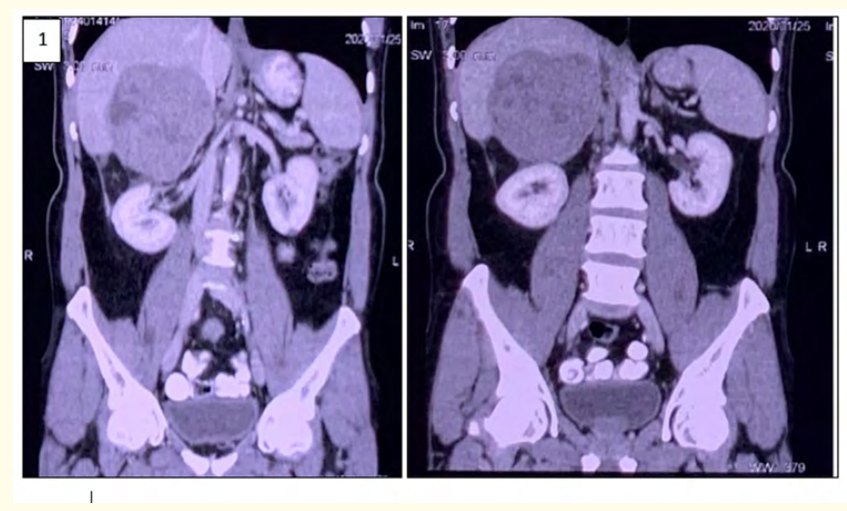

Figure 1: CECT scan coronal images shows well defined enhancing mass in the right supra renal area of size 9.7 x $8.4 \mathrm{cms}$.

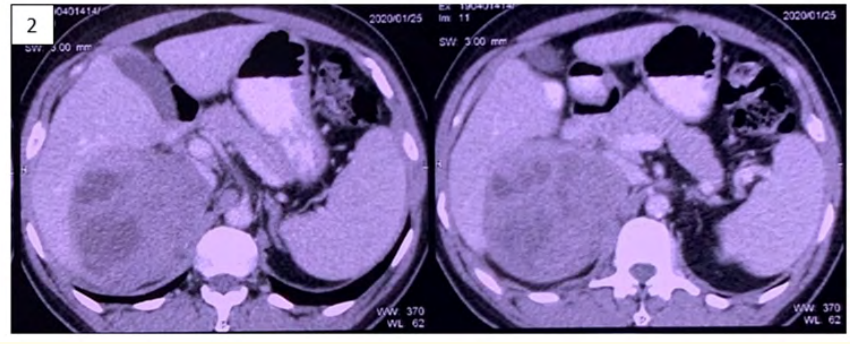

Figure 2: CECT scan axial images shows large right supra renal mass causing displacement of IVC.

Patient underwent evaluation for serum cortisol levels and 24hour urinary free metanephrines, which were within normal limits. Patient underwent further workup and taken for surgery after anaesthesiologist clearance. Right laparoscopic adrenalectomy was planned. Intraoperatively right adrenal gland was normal, and the tumour was seen arising from the confluence of right adrenal vein with IVC. Adrenal vein was dilated and engorged. Laparoscopy was converted to an open laparotomy with enbloc excision of mass with cuff of IVC lateral wall and adrenalectomy, followed by reconstruction of IVC rent with 4-0 size prolene suture (Figure 3, 4, 5, $6 \mathrm{a}$ and $6 \mathrm{~b})$. Right kidney was preserved. Intra operative and postoperative course was uneventful.

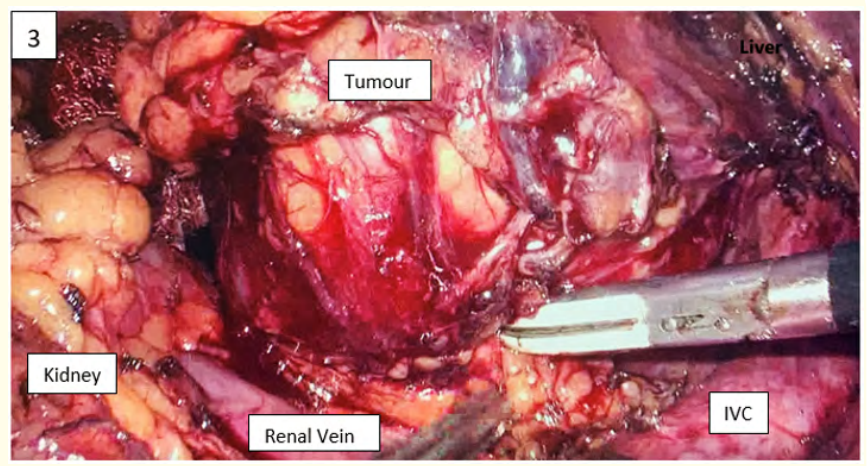

Figure 3: Laparoscopic dissection of mass showing the origin of mass from the lateral wall of IVC. 


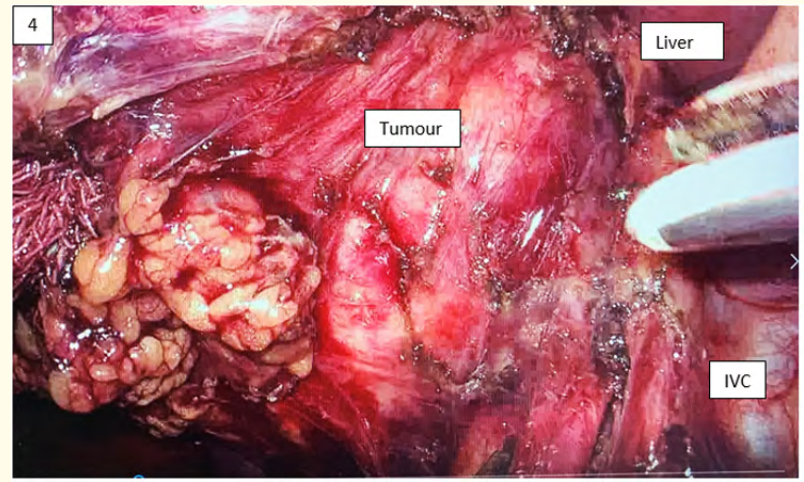

Figure 4: Laparoscopic dissection of mass showing the origin of mass from the lateral wall of IVC.

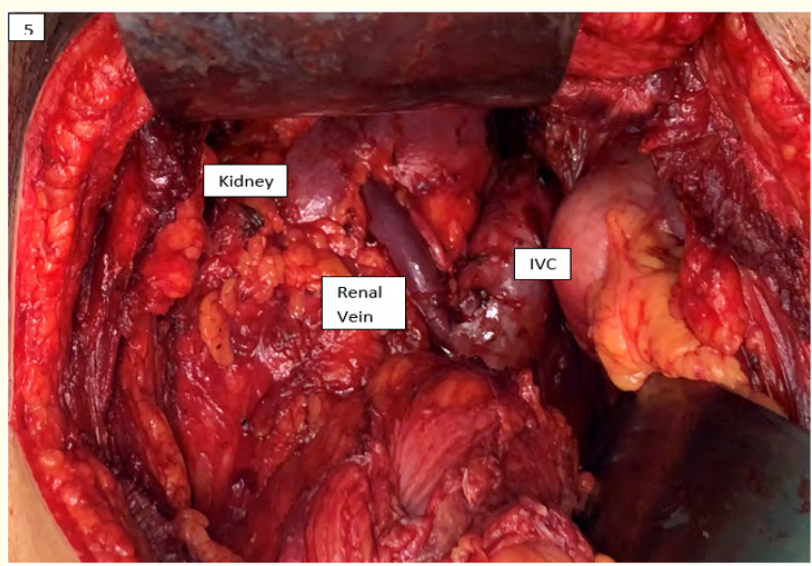

Figure 5: After complete en-bloc excision of tumour, photograph showing right kidney, renal vein and repaired IVC rent.
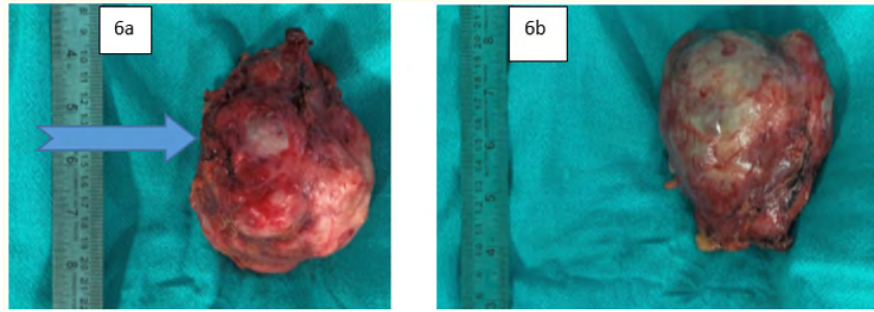

Figure 6: Specimen of IVC Leiomyosarcoma showing the cuff of IVC wall (arrow).
Grossly the tumour had a bosselated smooth outer surface with grey-white nodular whorled cut surface with foci of haemorrhage and necrosis. Compressed normal adrenal gland was identified.

Microscopy showed spindle cells in long interlacing bundles and fascicles with showing abundant eosinophilic cytoplasm, hyperchromatic nuclei with moderate pleomorphism. Brisk mitosis (8-10 per high power field) was noted (Figure $7 \mathrm{a}$ and $7 \mathrm{~b}$ ).
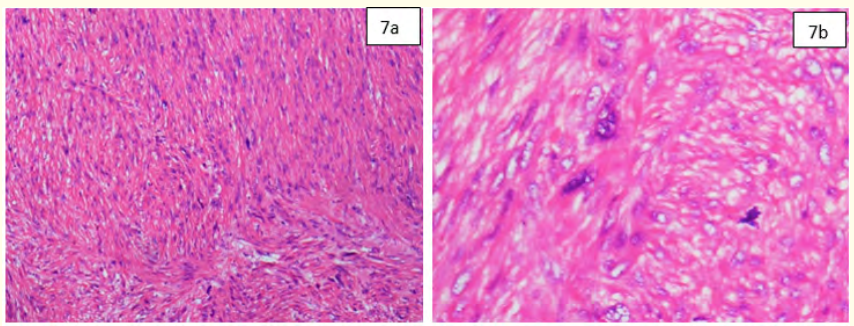

Figure 7: H\&E 100x showing spindle cells with nuclear pleomorphism. H\&E 400x showing spindle cells cells with atypical mitosis.

Histopathology report shows IVC leiomyosarcoma of $11 \mathrm{~cm}$ in greatest dimension with invasion of adrenal vein. Lympho vascular invasion was noted. Adrenal gland was free of tumour and margins of resection were free of tumour.

Immunohistochemistry was positive for SMA and caldesmon and negative for melan-A suggestive of leiomyosarcoma (Figure 8a and $8 \mathrm{~b})$.
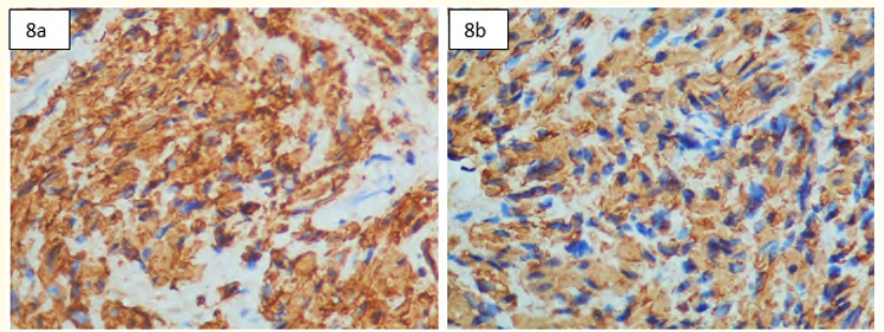

Figure 8: IHC Caldesmon 400x shows cytoplasmic positivity. IHC SMA 400x shows cytoplasmic positivity. 
Patient was discharged on post-operative day 7. No adjuvant therapy was given, and he was on regular follow-up for the past 1 year with no local recurrence or distant metastasis.

\section{Discussion}

LMS is not an uncommon soft tissue sarcoma in adults [4]. This tumour may arise from any structure or organ containing smooth muscle [5]. Retroperitoneum is one of the commoner sites of abdominal LMS [5]. Vascular leiomyosarcomas are exceedingly rare, representing less than $2 \%$ of all LMS [6]. Leiomyosarcoma of adrenal gland is an extremely rare tumour and is thought to originate from the main adrenal vein or its tributaries [7]. It may be difficult to differentiate between Leiomyosarcoma arising from the IVC and retroperitoneal sarcoma invading the IVC [8].

IVC LMS originates from the smooth muscle cells of vessel wall and its growth pattern may be intra-luminal or extra-luminal, with possibility of invasion of adjacent structures [9]. In intraluminal type of LMS, IVC lumen is dilated and filled with mass [10]. In extra luminal type of LMS, the primary tumour of the IVC grows exophytic, which is often misdiagnosed as tumour of the adjacent organs like adrenal gland mass or sarcoma arising from retroperitoneum with direct invasion into IVC [11].

Abdominal cavity has large room for growth of sarcoma without causing many symptoms or clinical signs to the patient at initial stages of disease [12]. Tumour size is one of the important prognostic markers for survival [5]. 5-year survival rates were around $30 \%$ to $53 \%$ in patients who underwent complete resection of tumour with negative margins [13].

En bloc resection of tumour with clear surgical margin along with removal of regional lymph nodes and followed by IVC reconstruction with or without vascular grafts is the best management in vascular LMS [14]. If the patient has metastatic disease or extensive local invasion or unfit for surgery or anaesthesia, IVC leiomyosarcoma may require a combination of radiation therapy and chemotherapy prior [15]. However, if intra-operatively, complete resection of tumour is not possible, debulking of tumour followed by local radiation therapy is a good palliative treatment option [16]. Chemotherapy with gemcitabine and docetaxel were used in the treatment of LMS [17].

\section{Conclusion}

Due to rarity, extra luminal leiomyosarcomas of the inferior vena cava still pose a challenge to surgeons in diagnosis and treatment especially when presenting as supra renal masses. Preoperative evaluation should include imaging to determine the exact site of origin and to differentiate from tumors arising from adjacent organs. Thorough clinical, radiological, and biochemical correlation is necessary for accurate preoperative diagnosis and to avoid untoward intraoperative complications. First-line treatment for LMS of adrenal vein or IVC is radical resection of the tumour with negative margins, which offers the best survival rate.

\section{Conflict of Interests}

The authors have no conflict of interest to declare.

\section{Bibliography}

1. Keiffer E., et al. "Leiomyosarcoma of the Inferior Vena Cava: Experience in 22 Cases". Annals of Surgery 244.2 (2006): 289295.

2. Singh D., et al. "A rare case of primary leiomyosarcoma of the inferior vena cava with an intra- and extravascular component". Indian Journal of Vascular and Endovascular Surgery 6 (2019): 315-319.

3. Lack EE., et al. "Primary leiomyosarcoma of adrenal gland. Case report with immunohistochemical and ultrastructural study". The American Journal of Surgical Pathology 15.9 (1991): 899-905.

4. Serrano Cesar and George Suzanne. "Leiomyosarcoma". Hematology/Oncology Clinics of North America 27.5 (2013): 957974.

5. Nascif RL., et al. "Leiomyosarcoma of the inferior vena cava: case report". Radiologia Brasileira 47.6 (2014): 384-386.

6. Dzsinich C., et al. "Primary venous leiomyosarcoma: a rare but lethal disease". Journal of Vascular Surgery 15 (1992): 595603. 
7. Thamboo TP., et al. "Adrenal leiomyosarcoma: a case report and literature review". Pathology 35 (2003): 47-49.

8. McClain KL., et al. "Association of Epstein-Barr virus with leiomyosarcoma in young people with AIDS". New England Journal of Medicine 332 (1995): 19.

9. Iliana B., et al. “Case 257: Leiomyosarcoma of the Inferior Vena Cava”. Radiology 288.3 (2018): 901-908.

10. Mankin HJ., et al. "Leiomyosarcoma of somatic soft tissues". Clinical Orthopaedics and Related Research 421 (2004): 225231.

11. Bednarova I., et al. "Case 257: Leiomyosarcoma of the Inferior Vena Cava”. Radiology 288.3 (2018): 901-908.

12. Sessa B., et al. "Imaging of leiomyosarcoma of the inferior vena cava: comparison of 2 cases and review of the literature". Cancer Imaging 10 (20210): 80-84.

13. Dew J., et al. "Leiomyosarcoma of the inferior vena cava: surgical management and clinical results". American Surgery 71 (2005): 497-501.

14. De Saint Aubain Somerhausen N and Fletcher C. "Leiomyosarcoma of Soft Tissue in Children: Clinicopathologic analysis of 20 cases". American Journal of Surgical Pathology 23.7 (1999): 755.

15. Wang TS., et al. "Leiomyosarcoma of the adrenal vein: a novel approach to surgical resection". World Journal of Surgical Oncology 5 (2007): 109.

16. Hemant D., et al. "Primary leiomyosarcoma of inferior vena cava, a rare entity: imaging features". Australas Radiology 45.4 (2001): 448-451.

17. Hensley ML., et al. "Gemcitabine and docetaxel in patients with unresectable leiomyosarcoma: results of a phase II trial". Journal of Clinical Oncology 20.12 (2002): 2824-2831.

\section{Assets from publication with us}

- Prompt Acknowledgement after receiving the article

- Thorough Double blinded peer review

- Rapid Publication

- Issue of Publication Certificate

- High visibility of your Published work

Website: www.actascientific.com/

Submit Article: www.actascientific.com/submission.php

Email us: editor@actascientific.com

Contact us: +919182824667 